\title{
Le « travail de la maladie » revisité par la psycho-oncologie
}

\author{
The "work of illness" revised by French psycho-oncologists
}

\author{
M.-F. Bacqué \\ (C) Springer-Verlag France 2013
}

La prise en charge des malades atteints de cancer se complète et se diversifie régulièrement. Elle s'enrichit aujourd'hui d'activités physiques, y compris à l'hôpital. Cette vision se veut intégrative : l'être humain n'est plus un corps et une âme, il devient une individualité psychosomatique en rééquilibrage permanent.

\section{Vertus de l'activité physique chez l'enfant atteint de cancer}

La pratique d'une activité physique a des vertus insoupçonnées dans le domaine du cancer. Jusqu'à il y a encore une trentaine d'années, le malade atteint de cancer se couchait dès le début des traitements et était censé se « reposer ». Aujourd'hui, les résultats de la pratique régulière d'un sport (depuis au moins six mois) sont édifiants : chez les femmes suivies pour un cancer du sein, l'activité physique diminue de moitié le risque de rechute [1].

La pratique d'un sport est donc hautement recommandée chez l'adulte, malade ou non. Mais qu'en est-il pour l'enfant atteint d'un cancer?

Sylvie Scaon et al. décrivent une expérience d'activité physique adaptée au CHRU de Clocheville, à Tours. Les enfants ayant bénéficié de l'APA sont âgés de 18 mois à plus de 14 ans, avec une proportion plus importante d'enfants en dessous de 6 ans. Que pratiquent-ils dans le service?

Selon l'âge, cela va des exercices de motricité fine jusqu'à de véritables compétitions au ping-pong ou au babyfoot. Ce qui change ici, ce ne sont pas tant les effets au long terme de l'activité sur les enfants, mais c'est surtout le vécu des parents. Satisfaits d'investir leur enfant autrement que comme un petit malade fragilisé, ils sont heureux de le voir reprendre des couleurs, retrouver du plaisir dans le mouvement, installer une dynamique d'échange avec les autres. Enfin, l'activité sportive témoigne, pour la plupart des

M.-F. Bacqué $(\square)$

Rédactrice en chef de Psycho-oncologie,

université de Strasbourg, France

e-mail : mfbacque@club-internet.fr parents, d'une amélioration de l'état de l'enfant qui peut, à l'intérieur même de la structure soignante, s'offrir le « risque » de bouger. En effet, les parents n'oseraient peutêtre pas, hors contexte, proposer à leur enfant la stimulation nécessaire pour sortir de l'immobilisme parfois dépressiogène de la maladie. Grâce à la proposition d'activité physique, même en secteur protégé, les relations se détendent et améliorent aussi l'état physique de l'enfant.

\section{Animation d'inspiration psychanalytique d'un groupe de parents dont l'enfant a un cancer}

Dorella Scarponi nous propose, de Bologne, un autre type d'expérience avec les parents d'enfants atteints de cancer. Elle fait le point, avec sa collègue, sur dix années d'animation de groupes de parents. Trois expériences différentes les amènent à la conclusion que le problème majeur est surtout lié à la discontinuité des allées-venues des parents, tandis que d'autres groupes sont envahis par la rage, la colère contre l'institution ou les lamentations et l'angoisse de la mort des enfants. Certains parents peuvent devenir coleaders des groupes et favoriser la confiance dans les animateurs. En revanche, la présence de l'oncologue dans le groupe transforme le processus en psychoéducation : les parents, au lieu d'échanger ou de partager des expériences, neutralisent leurs émotions pour s'adresser au médecin et le questionner sur la maladie et les traitements. Ce mécanisme de défense du groupe va tout à fait dans le sens du précédent : alors que les premières formes de réaction de la famille face au diagnostic de cancer sont l'agressivité et la rage, prédomine ensuite la tendance à arrêter le temps dans l'espace, dans une sorte de suspension sans histoire, la seule compatible avec l'« idée de la séparation ».

Les rôles des pères et des mères (italiens de Bologne) semblent distincts : les mères sont plus présentes et centrées sur la relation avec l'enfant, les pères gardent plus le silence et masquent leur douleur. Ils favorisent cependant plus le lien social et expriment des sentiments de honte et d'échec en rapport avec la place compétitive de la famille dans la 
société. Cette étude italienne n'est pas très éloignée des françaises. Elle se réfère à l'animation psychanalytique des petits groupes, à Bion [2], à Foulkes [3], mais aussi au travail de Neri [4] qui exerce à Rome. Les particularités du groupe de parents conjuguées à la problématique de l'enfant malade mériteraient tout un numéro de Psycho-oncologie. Cet article nous permet toutefois de constater le vécu habituel du groupe dans l'adversité : fantasme d'omnipotence ou de groupe vide, projection sur l'extérieur, recherche d'un thaumaturge, angoisse de mort aboutissant parfois au blocage de la pensée. Ces fantasmes sont, en l'absence de lieu d'expression, retenus de façon mortifère ou, au contraire, délivrés à l'extérieur de façon massive et non transformée. Tout cela ne laisse cependant pas indemnes les thérapeutes, c'est pourquoi une formation de qualité, doublée d'une supervision, est plus qu'indispensable.

Les articles suivants traitent de la maladie en fonction du genre.

\section{Charge psychique du cancer et genre}

Tout d'abord, nous avons le plaisir d'accueillir une équipe chinoise de Chengdu dans le Sichuan. Elle évoque la question du " fardeau », la charge psychique du cancer qui diffère, en Chine, selon les hommes et les femmes. Les facteurs culturels à l'origine de ces différences mériteraient d'être analysés finement par des sociologues et anthropologues chinois. Nous ne pouvons sans doute pas nous mettre à leur place, mais nous pouvons aisément projeter que, lorsqu'une femme est malade, outre la charge psychique du cancer, porteuse de l'image de la mortalité et du handicap potentiel, ce sont d'autres difficultés, liées aux rôles sociaux des femmes et des mères, qui s'additionnent.

Cet article pourra être comparé avec celui de Cécile Choimet et al. qui s'interroge sur l'opposition entre les représentations de la pathologie cancéreuse en général et celles de leur maladie personnelle, d'un échantillon de femmes ayant subi une tumorectomie et ses traitements complémentaires. D'un côté, on retrouve la représentation sociale du cancer dominante : celui-ci est associé à la mort, au fléau, au traumatisme, à la gravité, aux stigmates, à l'exclusion. Or, ces notions ne se retrouvent pas quand les interviewées parlent de leur maladie personnelle. Certaines vont d'ailleurs reprendre les avis de leurs médecins à leur compte : «J'ai eu de la chance... C'est un petit, gentil cancer... » Cette minimisation interroge les auteures de l'article quant aux possibilités d'accepter les soins de support chez ces femmes.

\section{Le « travail de la maladie » revisité}

Cécile Charles et ses collègues proposent de reprendre le concept de « travail de la maladie » pour caractériser la souf- france exprimée par les patients selon les trois niveaux identifiés par Jean-Louis Pedinielli (effraction, limitation de la réalisation du désir, mise en cause de l'identité). Il s'agirait d'aider au repérage puis à l'orientation des patients les plus vulnérables. Dans un second temps, la recherche et l'écoute des représentations associées à l'atteinte corporelle, au traitement et à la maladie constitueraient la voie la plus directe pour mieux comprendre ce qui met à mal la subjectivité du patient.

L'article de Rachel Ferrere et Jaqueline Wendland traite aussi de ces croisements de représentations sociales et individuelles. Elles évoquent ces cas rares où le cancer vient rencontrer la maternité. Ici aussi, on observe un système d'influence réciproque entre deux dynamiques psychiques antagonistes : l'ajustement à la maladie et le processus de maternalité. Si chaque année, en France, 350 à 750 femmes présentent un cancer du sein pendant leur grossesse, ce faible effectif mérite un suivi particulier éthiquement d'une part, mais aussi pour compléter les études beaucoup plus larges sur la constitution de l'être-mère (la maternalité). Leurs deux cas cliniques montrent les mécanismes de défense qui permettent de mettre à distance la menace que représente la maladie afin de laisser émerger la préoccupation maternelle primaire. Parfois, c'est justement la maternalité qui rendra l'ajustement à la maladie difficile, lorsque les traitements seront à l'œuvre après la naissance du bébé.

\section{Ré-érotiser, par la poésie, l'image du corps après un cancer}

Alphonso Santarpia et ses collègues apportent un peu d'originalité dans ces situations extrêmes. Ils proposent un « protocole d'écriture poéticoérotique » (nommé SANTEL) qui permet la ré-érotisation de l'image du corps chez une femme ayant vécu un cancer.

Le matériel est composé d'une liste de phrases à caractères poétique et érotique à choisir, d'un texte à trous à remplir, d'amorces de phrases ciblées sur le corps et enfin d'un poème libre. Dans un cas clinique, ils démontrent qu'une reconstruction perceptive et poétique de l'image érotique $\mathrm{du}$ corps peut donner lieu à une reprise des relations sensuelles dans un couple, alors même que la femme a subi une mastectomie. Les deux conjoints participent à ce recouvrement de leurs échanges sensuels, dans un discours un peu plus inducteur que l'écriture automatique, mais qui conduit clairement vers la liberté amoureuse retrouvée.

La poésie est composée de métaphores linguistiques élaborées, même si parfois elles prennent pour point de départ des images très crues. Ce qui frappe est alors la capacité de franchir plusieurs registres, de partir de la fange ou des miasmes morbides baudelairiens pour arriver à l'élévation la plus transcendantale. Jean-Louis Pujol et ses collègues travaillent sur la même thématique, au fond, puisqu'ils étudient la mise 
en représentations mentales de l'annonce du diagnostic de cancer. Dans trois observations cliniques approfondies, ils décrivent l'absence de représentation que produit la sidération de la pensée, l'utilisation de protoreprésentations simplistes circulant dans l'environnement social des patients, l'endossement par le malade d'un statut social lié au cancer, dans lequel il se glisse, mais sans aucunement se l'approprier. S'il n'y avait pas eu de traumatisme initial, un travail de symbolisation aurait sans doute été possible plus rapidement. Composé de représentations de mots, il croise les représentations sociales du cancer et celles propres à la personnalité de chaque patient. L'angoisse de mort engendrée par le choc de l'annonce ne permet pas cette mixation des affects. Les entretiens cliniques traduisent souvent cette mise en mots salutaire, la plupart du temps après coup, mais une fois cette étape franchie, le patient peut clairement employer son énergie psychique à intégrer ce qui lui arrive.

\section{Un projet de loi pour « l’aide médicale à mourir " au Québec}

La dernière contribution est un véritable plaidoyer de Mélanie Vachon, professeure de psychologie à l'UQAM, à réfléchir devant le projet d'une potentielle loi sur l'euthanasie au Québec.

Ce projet de loi 52 légaliserait la pratique de l'euthanasie sous la forme d'une « aide médicale à mourir », et ce, en dépit d'importantes contestations formulées par les experts en soins palliatifs. Il y a en effet une forme de mauvaise foi à appeler l'euthanasie une « aide médicale à mourir », tandis que les soins palliatifs sont à leur tour qualifiés de « lieu où l'on aide à mourir ». Un glissement d'expression permet de joindre, en un même terme, les deux « écoles » tout à fait opposées que sont les proeuthanasies et les soins palliatifs. Il est intéressant, à cette occasion, de lire le collectif québécois de médecins du refus total de l'euthanasie http://refusmedical.blogspot.ca/p/le-collectif-de-medecins.html et de comparer ce texte avec celui envoyé au CCNE le 15 juillet 2013 par six médecins belges qui l'accusent ouvertement de mensonges à leur égard. D'un côté, le collectif des médecins du Québec écrit : « Nous n'acceptons pas que les médecins se voient confier le rôle de juges de l'éligibilité à la mort provoquée, pas plus que celui de techniciens de la mort provoquée. » Ils observent que « la dépénalisation de l'euthanasie augmente l'acceptation sociale à proposer la mort comme solution aux problèmes de douleur et souffrances de la vie. Elle crée un climat où des familles et des soignants pourraient porter un regard dévalorisant sur les malades qui ont choisi de vivre leur vie jusqu'à sa fin naturelle, entraînant des pressions psychologiques sur ces malades ». Ils insistent sur le besoin prioritaire qui demeure celui de l'amélioration de l'accessibilité et de la qualité des soins de santé offerts aux personnes qui souffrent de maladies terminales et aux personnes âgées ou handicapées. De l'autre, les médecins belges considèrent que l'euthanasie est vieille comme le monde et que si Hippocrate trouvait nécessaire d'interdire aux médecins, dans son serment, l'emploi du poison, c'est bien que la demande existait ! Les suicides récents d'Hugo Claus (écrivain) et de Christian de Duve (prix Nobel de médecine) semblent justifier, à leurs yeux, une forme « d'euthanasie humanitaire (sic), mort rapide, sans douleur, considérée comme un bienfait par l'intéressé ». Ils terminent en généralisant à toute la Belgique, qui se sent « fière d'avoir entendu cet appel »... L'article de Mélanie Vachon permet de sortir de l'ornière émotionnelle dans laquelle les discussions pro- et antieuthanasies poussent à s'embourber. Cependant, elle insiste sur les difficultés à légaliser puisque, dans les pays où c'est le cas, persistent toujours des euthanasies illégales (sans l'accord des patients). Les proeuthanasies clament que la légalisation de l'euthanasie s'inscrit dans la continuité du progrès médical ; or, ce progrès semble incohérent avec la philosophie des soins palliatifs. Y aurait-il donc deux médecines ? Il y a peut-être deux philosophies de la vulnérabilité ; avec, d'un côté, ceux qui prétendent que la souffrance et la mort n'ont plus de sens depuis la sécularisation des sociétés occidentales et ceux qui, au contraire, pensent qu'une éthique des soins en fin de vie « vise davantage un travail de prévention et de sensibilisation sur le rapport à la mort, à la dignité et à la vieillesse des sociétés contemporaines ». Cette conclusion, très argumentée de Mélanie Vachon, nous permet, grâce à l'exemple du Québec, de poursuivre notre propre débat en France. Le parcours est long, mais ne peut qu'être résolument interdisciplinaire et nourri d'autres expériences humaines.

\section{Références}

1. Sternfeld B, Weltzien E, Quesenberry CP, et al (2009) Physical activity and risk of recurrence and mortality in breast cancer survivors: finding from the LACE study. Cancer Epidemiol Biomarkers Prev 18:87-95

2. Bion WR (1961) Recherches sur les petits groupes. Paris PUF (traduction française, 1965)

3. Foulkes SH (1975) Group-Analytic Psychotherapy: Methods and Principles, London, Gordon and Breach

4. Neri C (1995) Le Groupe. Manuel de psychanalyse de groupe. Paris, Dunod (traduction française, 1997)

\section{Références électroniques}

5. Lettre ouverte au président et aux membres du Comité consultatif national d'éthique datée du 15/07/2013 et cosignée par Pr Distelmans, Dr Lossignol, Dr Englert, Dr Damas, Dr de Deyn, Dr Deron

6. Le collectif québécois de médecins du refus total de l'euthanasie. http://refusmedical.blogspot.ca/p/le-collectif-de-medecins.html 